\title{
CONCENTRATION CHANGES DUE TO CATHODIC PROTECTION*
}

\author{
P. J. Gellings \\ Technical University Twente, Department of Chemical Engineering, Laboratory for Inorganic \\ Chemistry and Materials Science, Enschede, Netherlands
}

\begin{abstract}
By solving the appropriate diffusion equations the concentration changes are calculated in the environment of underground structures protected cathodically. It is shown that these changes are negligible under all practical circumstances.
\end{abstract}

\section{INTRODUCTION}

DURING cathodic protection the concentrations of several substances, amongst others, those of $\mathrm{H}^{+}$and of $\mathrm{OH}^{-}$ions, change, due to the current flowing. ${ }^{1,2}$ In principle this may have undesirable consequences for the environment.

In this paper the solution of the differential equation for the concentrations as a function of time and position is presented for several important geometries: semiinfinite linear, cylindrical and spherical.

Substituting the appropriate numerical values of the different parameters shows that under all practical circumstances cathodic protection of underground structures produces negligible changes of the concentrations in the environment of the protected parts.

\section{BASIC EQUATIONS}

The electrode reaction is written as

$$
-\sum_{i} v_{i} X_{i}+\mathrm{n} e^{-}=0
$$

The concentration $c_{i}$ of the component $X_{i}$ is given by Fick's second law:

$$
\nabla^{2} c_{i}-\frac{1}{D_{i}} \frac{\partial c_{i}}{\partial t}=0
$$

This has to be solved subject to the boundary condition that at the surface of the conductor which is protected (and of course also at the surface of the anode):

$$
\left(\nabla c_{i}\right)_{\text {surface }}=-\frac{I v_{i}}{\mathrm{n} F D_{i}} \quad t>0
$$

*Manuscript received 5 November 1977; in amended form 19 December 1977. 
where $I$ is the current density. Furthermore at an infinite distance from the a node and the cathode we have the second boundary condition:

$$
c_{i}=c_{i}^{\circ} \quad t>0,
$$

where $c_{i}{ }^{\circ}$ is the original concentration of component $X_{i}$. Finally there is the initial condition:

$$
c_{i}=c_{i}{ }^{\circ} \quad t \leq 0, \text { all positions. }
$$

The set of equations (2)-(5) can be transformed in a more convenient form by introducing the relative concentration $u_{i}$ as:

$$
u_{i}=\left(c_{i}-c_{i}{ }^{0}\right) / c_{i}{ }^{\circ} .
$$

For semi-infinite linear diffusion, i.e. cathodic protection of an infinite flat plate the equations (2)-(5) become

$$
\begin{aligned}
& \frac{\partial^{2} u_{i}}{\partial x^{2}}-\frac{1}{D_{i}} \frac{\partial u_{i}}{\partial t}=0 \\
& \left(\frac{\partial u_{i}}{\partial x}\right)_{x=0}=-\frac{I v_{i}}{n F D_{i} c_{i}^{\circ}}=-A_{i} \\
& u_{i}(x=\infty)=0 \quad \text { all } t \\
& u_{i}(\text { all } x)=0 \quad t \leq 0
\end{aligned}
$$

For cylindrical diffusion, i.e. in the case of an infinitely long cylinder of radius $a$, such as in the cathodic protection of a pipeline, we obtain:

$$
\begin{aligned}
& \frac{\partial u^{2}}{\partial r^{2}}+\frac{1}{r} \frac{\partial u_{i}}{\partial r}-\frac{1}{D_{i}} \frac{\partial u_{i}}{\partial t}=0 \\
& \left(\frac{\partial u_{i}}{\partial r}\right)_{r=a}=-A_{i} \\
& u_{i}(r=\infty)=0 \quad \text { all } t \\
& u_{i}(r \geq a)=0 \quad t \leq 0 .
\end{aligned}
$$

For spherical diffusion, i.e. in the case of a sphere of radius $a$, which approximates to the case of cathodic protection of an underground tank, rewriting gives:

$$
\begin{aligned}
& \frac{\partial^{2} u_{i}}{\partial r^{2}}+\frac{2}{r} \cdot \frac{\partial u_{i}}{\partial r}-\frac{1}{D_{i}} \frac{\partial u_{i}}{\partial t}=0 \\
& \left(\frac{\partial u_{i}}{\partial r}\right)_{r=0}=-A_{i} \\
& u_{i}(r=\infty)=0 \text { all } t
\end{aligned}
$$




$$
u_{i}(r \geq a)=0 \quad t \leq 0
$$

\section{SOLUTION OF DIFFUSION EQUATIONS}

The application of the Laplace transformation ${ }^{3}$ presents a convenient way to solve the sets of equations (7)-(9). The Laplace transform of a function $v$ is denoted by $\bar{v}$ and $£$ is used as a symbolic notation for the Laplace transformation which is defined as:

$$
\mathfrak{f}(\nu)=\bar{v}=\int_{0}^{\infty} \mathrm{e}^{-p t} \mathrm{v}(x, t) \mathrm{d} t .
$$

Applying this to the equations (7)-(9) the solution $\bar{u}(x, p)$ can be obtained. From this we then obtain, applying the inverse transformation, symbolically written $\mathfrak{E}^{-1}$, the desired solution:

$$
u(x, t)=\mathfrak{f}^{-1}\{\bar{u}(x, p)\} .
$$

\section{Semi-infinite linear diffusion}

Applying equation (10) to the set of equations (7) gives:

$$
\begin{aligned}
& \frac{\mathrm{d}^{2} \bar{u}_{i}}{\mathrm{~d} x^{2}}-\frac{p}{D_{i}} \bar{u}_{i}=0 . \\
& \left(\frac{\mathrm{d} \bar{u}_{i}}{\mathrm{~d} x}\right)_{x=0}=-\frac{A_{i}}{p} \\
& \vec{u}_{i}=0 \quad x=\infty .
\end{aligned}
$$

Making use of the boundary condition of equation (12c) this gives as a general solution:

$$
\tilde{u}_{i}=P_{i} \exp \left\{-x\left(\frac{p}{D_{i}}\right)^{t}\right\}
$$

Applying the boundary condition of equation (12b) this gives

$$
P_{i}=A_{\mathrm{i}} D_{i}^{1 / 2} p^{-3 / 2} .
$$

Substituting this into equation (13) and using a table of inverse Laplace transforms ${ }^{3}$ directly gives:

$$
u_{\mathrm{i}}=2 A_{i} D_{i}^{\mathrm{i}}\left[\frac{t^{t}}{\pi^{\mathrm{t}}} \exp \left(-\frac{x^{2}}{4 D_{\mathrm{i}} t}\right)-\frac{x}{D_{i}^{\mathrm{t}}} \operatorname{erfc}\left\{x\left(D_{i} t^{-\mathrm{t}}\right\}\right] .\right.
$$

This function has its maximum at the protected surface, i.e. at $x=0$ where the concentration change will thus be largest. 
The value of this function at $x=0$ is:

$$
\left[u_{i}(x=0)\right]_{\mathrm{semi} \text { inf }}=\frac{2 I \mathrm{v}_{i} t^{\ddagger}}{\mathrm{n} F c_{i}{ }^{\circ}\left(\pi D_{i}\right)^{\natural}} .
$$

\section{Cylindrical diffusion}

Laplace-transformation of the set of equations (8) gives

$$
\begin{aligned}
& \frac{d^{2} \bar{u}_{i}}{\mathrm{~d} r^{2}}+\frac{1}{r} \frac{\mathrm{d} \bar{u}_{i}}{\mathrm{~d} i}-\frac{p}{D_{l}} \bar{u}_{i}=0 \\
& \left(\frac{\mathrm{d} \bar{u}_{i}}{\mathrm{~d} r}\right)_{r=a}=-\frac{A_{i}}{\rho} \\
& \bar{u}_{i}=0 \quad r=\infty .
\end{aligned}
$$

Equations of this type have been discussed by Carslaw and Jaeger. ${ }^{3}{ }^{4}$ The general solution of equation (17a), which is the Bessel differential equation of order zero, can be written as:

$$
\bar{u}_{i}=P_{i} K_{\mathrm{o}}\left(q_{i} r\right)+Q_{i} I_{\mathrm{o}}\left(q_{i} r\right)
$$

with

$$
q_{i}=\left(p / D_{i}\right)^{\frac{1}{t}}
$$

$K_{\mathrm{o}}$ and $I_{\mathrm{o}}$ are zeroth order modified Bessel functions. From the boundary condition of equation (17c) it is deduced that we must have $Q_{i}=0$. From the boundary condition of equation (17b) it follows that:

$$
P_{i}=A_{i} / q_{i} K_{1}\left(q_{i} a\right)
$$

so that, with equation (18), we obtain:

$$
\bar{u}_{i}=\frac{A_{i} K_{\mathrm{o}}\left(q_{i} r\right)}{p q_{i} K_{\mathrm{r}}\left(q_{i} a\right)}
$$

The inverse of this function cannot be written in closed form in known functions. It can be obtained as the integral: ${ }^{4}$

$$
\begin{aligned}
u_{l} & =\frac{2 A_{i}}{\pi} \int_{0}^{\infty}\left\{1-\exp \left(-D_{i} w^{2} t\right)\right\} \\
& \times \frac{Y_{\mathrm{o}}(w r) J_{1}(w a)-J_{\mathrm{o}}(w r) Y_{1}(w a)}{w^{2}\left[J_{1}^{2}(w a)+Y_{1}^{2}(w a)\right]} \mathrm{d} w .
\end{aligned}
$$


For not too large values of $D_{i} t / a^{2}$ this integral can be approximated by the expression:

$$
\begin{aligned}
u_{\mathrm{i}} & =2 A_{i}\left(\frac{D_{1} a t}{\pi r}\right)+\left\{\pi^{\ddagger} i \operatorname{erfc} \frac{r-a}{2\left(D_{l} t\right)^{\dagger}}-\right. \\
& \left.-\frac{(3 r+a)\left(\pi D_{i} t\right)^{t}}{4 a r} i^{2} \operatorname{erfc} \frac{r-a}{2\left(D_{i} t\right)^{\ddagger}}+\ldots\right\} .
\end{aligned}
$$

This function has its maximum for $r=a$, i.e. at the surface of the cylindrical conductor. There its value can be written:

$$
u_{l}(r=a)=2 A_{i}\left(\frac{D_{i} t}{\pi}\right)^{t}\left\{1-0.25 \frac{\left(\pi D_{i} t\right)^{t}}{a}\right\}
$$

or

$$
u_{i}(r=a)=\left[u_{i}(x=0)\right]_{\text {semi inf }}\left\{1-0.443 \frac{\left(D_{i} t\right)^{\mathbf{t}}}{a}\right\}
$$

where we have used the result given in equation (16).

Spherical diffusion

Application of the Laplace transformation to the set of equations (8) gives:

$$
\begin{aligned}
& \frac{\mathrm{d}^{2} \bar{u}_{i}}{\mathrm{~d} r^{2}}+\frac{2}{r} \frac{\mathrm{d} \bar{u}_{i}}{\mathrm{~d} r}-\frac{p}{D_{i}} \bar{u}_{i}=0 \\
& \left(\frac{\mathrm{d} \bar{u}_{i}}{\mathrm{~d} r}\right)_{r=a}=-\frac{A_{i}}{p} \\
& \bar{u}_{i}=0 \quad r=\infty .
\end{aligned}
$$

The first of these equations can be written in the form:

$$
\frac{\mathrm{d}^{2}\left(r \bar{u}_{i}\right)}{\mathrm{d} r^{2}}-\frac{p}{D_{i}}\left(r \bar{u}_{i}\right)=0,
$$

which has the same form as equation (12).

From this we directly obtain, using the boundary condition of equation (26c):

$$
r \bar{u}_{i}=P_{i} \mathrm{e}^{-q_{r} r},
$$

where $q_{i}$ is given by equation (19).

The boundary condition of equation (26b) then leads to:

$$
\left(\frac{\mathrm{d} \bar{u}_{i}}{\mathrm{~d} r}\right)_{r=a}=-P_{l}\left(\frac{1}{a^{2}}+\frac{q_{l}}{a}\right)^{\mathrm{e}^{-q_{\imath} r}}=-\frac{A_{l}}{p}
$$


from which we obtain

$$
P_{i}=\frac{A_{i} a \mathrm{e}^{a q_{1}}}{p\left(q_{i}+1 / a\right)}
$$

and finally

$$
\bar{u}_{i}=\frac{A_{i} a \mathrm{e}-(r-a) q_{1}}{z p\left(q_{i}+1 / a\right)}
$$

Using formula 14 of Appendix $\mathrm{V}$ of Carslaw and $\mathrm{Jaeger}^{3}$ then gives:

$$
\begin{aligned}
& u_{i}=\frac{A_{i} a^{2}}{r}\left[\operatorname{erfc} \frac{r-a}{2\left(D_{i} t\right)^{\frac{1}{t}}}-\right. \\
& \left.-\exp \left\{\frac{r-a}{a}+\frac{D_{i} t}{a^{2}}\right\} \operatorname{erfc}\left\{\frac{r-a}{2\left(D_{i} t\right)^{\frac{1}{2}}}+\frac{\left(D_{i} t\right)^{\frac{1}{t}}}{a}\right\}\right] .
\end{aligned}
$$

For $r=a$, i.e. at the surface of the sphere, this function has a maximum. For this we obtain:

$$
u_{i}=A_{i} a\left\{1-\exp \left(\frac{D_{i} t}{a^{2}}\right) \operatorname{erfc} \frac{\left(D_{i} t\right)^{t}}{a}\right\} .
$$

For not too large values of $D_{i} t / a^{2}$ this can be approximated by:

$$
u_{i}=2 A_{i}\left(\frac{D_{i} t}{\pi}\right)^{t}\left\{1-1.220 \frac{\left(D_{i} t\right)^{t}}{a}\right\}
$$

or

$$
u_{i}=\left[u_{i}(x=0)\right]_{\mathrm{semi} i n f}\left\{1-1.220 \frac{\left(D_{i} t\right)^{\frac{1}{2}}}{a}\right\},
$$

by using the result of equation (16).

\section{NUMERICAL RESULTS}

The solutions for the concentration change $\Delta c_{i}=u_{i} \times c_{i}^{\circ}$ given by equations (16), (25) and (34) can be written in the general form:

$$
\Delta c_{i}=\frac{2 I \nu_{i} l^{\ddagger}}{\mathrm{n} F\left(\pi D_{i}\right)^{\natural}}\left\{1-\alpha \frac{\left(D_{i} t\right)^{\mathbf{t}}}{a}\right\},
$$

with

$\alpha=0$ : semi-infinite linear diffusion;

$\alpha=0.443$ : cylindrical diffusion around a cylinder with radius $a$;

$\alpha=1.220$ : spherical diffusion around a sphere with radius $a$. 
From this equation it is clear that in principle no stationary state will be reached in any of these cases, the concentration change increasing monotonously with time. However, due to convection and motion of the electrolyte these concentration differences will be continuously levelled out. Published figures for liquid motion in the ground ${ }^{5,6}$ show this to be of the order of millimeters to centimeters per hour. From this we can take as the maximum time $t$ in equation (35) one week, i.e. approximately $6 \times 10^{5} \mathrm{~s}$. For our calculations we further take $D_{i}=10^{-6} \mathrm{~cm}^{2} \mathrm{sec}^{-1}$, i.e. one tenth of that found in an aqueous solution and $v_{i}=n$.

The current requirements for cathodic protection vary widely depending on the type of soil, state of coating, etc. They may be anywhere between 0.001 and $10 \mathrm{~mA}$ per square meter of protected surface. ${ }^{7,8}$ For the conditions we assume in our estimates of $t_{\max }$ and $D_{i}$, i.e. for a not too wet soil, we take $I=1 \mathrm{mAm}^{-2}=10^{-7} \mathrm{Acm}^{2}$ as the maximum required protection current density.

Inserting these figures in equation (35) gives:

at the surface.

$$
\left(\Delta c_{i}\right)_{\text {semi-inf }}=9 \times 10^{-4} \mathrm{mol.1} \mathrm{l}^{-1}
$$

For a cylinder of dia. $40 \mathrm{~cm}$ this has to be multiplied by 0.983 , for a sphere of dia. $200 \mathrm{~cm}$ by 0.995 .

From equation (15) it can be calculated that at a distance of $5 \mathrm{~cm}$ from a plane electrode the concentration change is, under the conditions considered here, only about a hundredth from that at the suiface.

\section{DISCUSSION}

The result given by equation (36), calculated for the least favourable conditions likely to be met in practice, shows that even then the change in concentration is extremely small. In practice, the concentration changes will, moreover, nearly always be much smaller.

This shows that no adverse effects on the environment have to be expected from concentration changes due to cathodic protection. In faci solid corrosion products formed when cathodic protection is not applied may well be a greater nuisance. Even more serious is contamination of the soil due to leaks in unprotected pipelines or tanks which may cause very heavy damage to the environment. Thus cathodic protection of underground pipelines and tanks containing oil products is prescribed by law in many countries in particular in agricultural soil or in regions where the groundwater is used for the production of drinking-water.

\section{REFERENCES}

1. H. J. ENGell and P. ForchHammer, Corros. Sci. 5, 479 (1965).

2. P. J. Gellings and H. C. Ekama, Corros. Sci. 15, 529 (1975).

3. H. S. Carslaw and J. C. JAeger, Conduction of Heat in Solids, 2nd Edn. Oxford University Press, London (1959).

4. H. S. Carslaw and J. C. JAeger, Proc. London Math. Soc. 46, 361 (1940).

5. G. M. FAIR and J. CH. GeYer, Water Supply and Waste-water Disposal. Wiley, New York (1963).

6. R. K. Linsley, JR., M. A. Kohler and J. L. H. Paulhaus, Hydrology for Engineers, 2nd Edn. McGraw-Hill, New York (1975).

7. J. H. MORGaN, Cathodic Protection, Leonard Hill, London (1959).

8. L. L. ShreIR (Ed.), Corrosion, Vol. 2, Corrosion Control, 2nd Edn. Newnes-Butterworths, London (1976). 